\title{
Depression as a Disorder of Consciousness
}

\author{
Cecily Whiteley
}

To appear in the British Journal for the Philosophy of Science.

\begin{abstract}
First-person reports of Major Depressive Disorder reveal that when an individual becomes depressed a profound change or 'shift' to one's conscious experience occurs. The depressed person reports that something fundamental to their experience has been disturbed or shifted; a change associated with the common but elusive claim that when depressed one finds oneself in a 'different world' detached from reality and other people. Existing attempts to utilise these phenomenological observations in a psychiatric context are challenged by the fact that this experiential 'shift' characteristic of depression appears mysterious and resists analysis in scientific terms. This paper offers a way out of this predicament. The hypothesis proposed is that when an individual becomes depressed, the individual departs from a state of ordinary wakeful consciousness and enters a distinctive global state of consciousness akin to dreaming and the psychedelic state. After unpacking and motivating this hypothesis in the context of research in consciousness science, I outline two of its important implications for the neurobiology of depression and psychedelic psychiatry. The upshot is a promising and conceptually well-motivated hypothesis about depression which is apt for empirical uptake and development.
\end{abstract}

$1 \quad$ What is Depression?

2 Depression in a Clinical Context

3 The Phenomenology of Depression

$4 \quad$ What is a Global State of Consciousness?

5 The Depressive State of Consciousness

6 Empirical Evidence and Application

6.1 Global State and MDD Research: A Reciprocal Relationship

6.2. Psychedelic Psychiatry

7 Conclusion

\section{What is Depression?}

Depression is of interest to philosophy of psychiatry for a variety of reasons. Most prominently, depression (and the clinical category 'Major Depressive Disorder') is useful as a typical example of a 
diagnostic category or kind. A central question within philosophy of psychiatry in the last twenty years has been whether psychiatry's diagnostic categories, of which depression is a paradigmatic instance, carve nature at its joints in the same way as physical or chemical categories like 'electron' or 'hydrogen' do, such as to grant psychiatry scientific legitimacy (Beebee and Sabbarton-Leary [2010], Tsuo [2013]; [2016], Kincaid and Sullivan [2014], Tekin [2016] Tabb [2019]). This popular question of psychiatry's natural kind status can be distinguished from at least one further question concerning the nature of depression as a mental disorder - namely, what is depression and what distinguishes it from other mental disorders? (Kingma [2013]) - which the numerous answers to the question of whether depression is a natural kind underdetermine ${ }^{1}$.

Existing answers to this question in the philosophy of psychiatry seek to equate depression either with its current diagnostic symptoms or with their causal aetiology. However, the sufficiency of these descriptivist and causal answers to the question What is depression? have recently been challenged by phenomenological analyses of depression (primarily, Ratcliffe [2015]) which suggest that these views are currently incomplete insofar as they fail to capture something essential to what it is to be depressed; something which goes missing if one focuses solely on the existing diagnostic effects of depression or their constitutive causes. This is the observation, as revealed by extensive first-person reports of Major Depressive Disorder ('MDD'), that when an individual becomes depressed a distinctive phenomenological change occurs. The depressed individual reports that a 'profound shift' has occurred with respect to the way she is 'rooted' in the world or in reality; a distinctive change which is connected to the elusive sense in which depression involves a feeling of detachment from the world and from other people. The distinctive experiential changes which occur when individuals become depressed ought to be taken seriously as an important source of data for psychiatric research. However, the empirical utility of this phenomenological observation for the diagnosis and treatment of depression is severely limited by the fact that it is currently unclear how to elucidate and study this experiential shift empirically. The reports just mentioned raise the question of what this fundamental change is, such that it can be empirically studied and operationalised. This thought is exacerbated by Ratcliffe's endorsement of a phenomenological theory of depression as an 'existential shift', motivated by these phenomenological observations, which rejects the possibility that a full understanding of depression can be attained from a scientific perspective.

This paper outlines and motivates a new hypothesis about the nature of depression which, in a departure from Ratcliffe, accounts for and explains the distinctive phenomenology of depression in naturalistic terms. This is the hypothesis that when an individual becomes depressed the individual withdraws from a state of ordinary wakeful consciousness and enters a distinctive global state of consciousness. On this view, the changes an individual undergoes when she becomes depressed are

1 Compare, for example, how the claim that the biological species Homo Sapien is a natural kind, as opposed to an individual (Slater [2013]), underdetermines a number of more specific theses about what it is to be human. 
profound in the sense that they resemble (being changes of the same kind) the sorts of changes to her experience that occur when she departs from waking consciousness and starts dreaming, or when she enters a psychedelic state. I shall argue that this claim, which has become available only recently due to advances in consciousness science, captures and explains the distinctive experiential 'shift' revealed by Ratcliffe's phenomenological analysis of first-person reports of depression.

The plan for the paper is as follows. Sections 2 and $\mathbf{3}$ outline the two views of depression which emerge from current clinical literature on MDD and explain how Ratcliffe's phenomenological analysis of depression - as involving an experiential shift - suggests that these views are currently insufficient or incomplete. The key claim here is that there is a level of explanation essential or necessary to understanding what goes on in depression which is left out by views which focus solely on the existing diagnostic symptoms or their underlying constitutive causes (Kendler and Gyngell [2020]). This leaves us with a puzzle concerning how to elucidate this level of explanation in a way which is amenable to scientific investigation. Drawing on recent work in consciousness science Section 4 outlines a prominent account of global states on which global states of consciousness are multi-dimensional states which regulate the sorts of capacities that are online or available for a creature. In Section 5, I use this multi-dimensional view to motivate and flesh out a new hypothesis about depression according to which being depressed involves a change to an individual's global state of consciousness. This can be viewed, I argue, either as supplementing current views of depression, or as offering a new middle ground between existing accounts. Drawing on Ratcliffe's work, here I offer a more specific characterisation of this 'depressive state of consciousness', and explain how this proposal can be rendered compatible with the plausible claims that a person who is depressed remains awake and retains the capacity to dream. I argue these observations can be accommodated if one views depression as an extended temporal process with depressive global states as temporal parts. Philosophers and psychiatrists are not only interested in understanding what depression is and what it involves, but also in a host of further questions which relate to its neurological basis and treatment. I conclude in Section 6 by outlining two salient implications of the new hypothesis which shed light on these clinically pressing questions. This includes a suggestion for future empirical research which can shed light on the viability of the claim outlined in this paper, and move research forward.

\section{Depression in a Clinical Context}

The obvious starting place when answering the question What is depression? is the clinical taxonomies in which the diagnostic category MDD is listed ${ }^{2}$. According to the latest version of the

\footnotetext{
2 One might object to this on the basis of that mental disorders like MDD are too causally heterogenous to be of use to psychiatric research (See Hyman [2010], Lilienfeld [2014], Insel [2014], Hoffman and Zachar [2017], Tabb [2015], [2019]). While the hypothesis I motivate later may well have implications for this debate with respect to the category MDD and the Research Domain Criteria (RDoC), I set these issues aside here. Instead, I work with the mostly uncontroversial assumption that there is a distinctive phenomenon (or group of phenomena) tracked by the category MDD, although this may require substantial revision in accordance with the RDoCs aims at a later stage.
} 
Diagnostic Statistical Manual (the DSM-5; 2013), Major Depressive Disorder is characterised by at least one discrete depressive episode lasting no less than two weeks. As exemplified by mental disorders generally, a depressive episode is diagnosed polythetically, on the basis of the presence of a group of common symptoms only some of which are necessary for a diagnosis (Fellowes [2021]). Thus in addition to the presence of two core symptoms - a 'depressed mood' and a 'markedly diminished interest or pleasure in all, or all most all, activities' - the DSM states that at least four of the following symptoms must be present to warrant a diagnosis of Major Depressive Disorder 3 :

- Considerable weight loss or weight gain when not dieting, or decrease or increase in appetite,

- Sleep disturbances resulting in insomnia or hypersomnia,

- Fatigue or loss or energy,

- Feelings of worthlessness or guilt, which may be delusional; that is, not merely self-reproach or guilt about being sick,

- Diminished ability to think or concentrate, or indecisiveness,

- Recurrent thoughts of death (not merely dying), recurrent suicidal ideation without a specific plan; the individual has made a suicide attempt or specific plan for committing suicide.

Reflection on these diagnostic criteria gives rise to two standard views of depression found in the empirical and philosophical literature (Radden [2003]). According to 'ontological descriptivism', associated with the DSM-5, depression as a mental disorder can be equated or identified with the characteristic signs and symptoms of MDD just listed (Kendler et al. [2010])4. This stands in contrast to a 'causal' view of depression which moves away from an understanding of depression in terms of its symptoms to the causes of these symptoms 5 . This is compatible with a number of different views about the appropriate level of explanation required here (Murphy [2010]). That is, it is compatible with both a biomedical view which stipulates that mental disorders must be characterised and explained at a biological level (i.e in terms of genetic influence, molecular changes, brain dysfunction etc.) and with broader biopsychological models which leave room for a pluralistic, multi-levelled explanation 6 . On these causal views, MDD works in much the same way as a diagnosis of appendicitis: the

\footnotetext{
${ }^{3}$ In addition, it is stated that these symptoms must cause significant distress or impairment in socio-occupational contexts, and that symptoms better attributable to another medical condition (including other mental disorders or substance abuse) should not count towards a diagnosis. See also the ICD-11 [2020].

${ }^{4}$ This accommodates both a 'minimal descriptivist view' which identifies depression with it's 'key symptoms' such as depressed mood (Ventham [2019], Wong [2016]), and a more demanding 'threshold' conception on which depression is identified with these core symptoms only when they are present over a long period of time or at a high level of severity (Maj [2011], discussed in Tully [2018]).

5 This is concurrent with the way in which diagnostic categories are used in medicine more generally. Here, for example, a diagnosis of appendicitis explains why the patient has abdominal pain by conveying information about what is causing it (Haung [2016]).
}

${ }^{6}$ Murphy [2013], c.f. Davies, Savulescu, Roache and Loebel [2020] and Bolton and Gillett [2019)]. 
diagnostic category refers to the hidden cause or explanation, at whatever level, of why an individual exhibits the range of characteristic symptoms? ${ }^{7}$.

\section{The Phenomenology of Depression}

Part of the appeal of ontological descriptivism is that when it comes to mental illnesses, the mental or psychological effects implicated seem to be constitutively connected to these disorders in a way which distinguishes them somewhat from somatic illnesses ${ }^{8}$. This idea is reflected in the fact that the majority of MDD's diagnostic criteria make reference to changes in a patient's conscious mental life for example, feelings of worthlessness, a depressed mood, recurrent thoughts of death, and an inability to think and concentrate. This sort of claim suggests that in order to improve our understanding of depression psychiatrists ought to collect more detailed accounts of the changes to one's mental life which occur when one becomes depressed. This task has been taken up recently by Ratcliffe who, in his book 'Experiences of Depression' ([2015]) offers a comprehensive analysis of what it is like to be depressed, with the aim of producing more fine-grained criteria which can be employed in clinical contexts to reduce false diagnoses. Motivated by a comprehensive analysis of first person reports of depression taken from both depression memoirs and an online questionnaire (SANE [2011]; Appendix [2015]) Ratcliffe observes that a diagnosis of MDD in fact tracks several salient (and often connected) changes to a subjects mental life, many of which are left out of, or brushed over, in the DSM-5's existing diagnostic criteria9:

- Changes to bodily resonance (feelings of fatigue, tiredness, lethargy, heaviness, sickness or nausea, loss of appetite, numbness).

- Feelings of hopelessness (in particular, the loss of an ability to hope simplicter ('existential hope') rather than feeling hopeless about something).

- Feelings of guilt and changes to one's self-narrative (feeling guilty about something specific, feeling guilty about something but not knowing what, feeling that one is guilty of something specific, feeling guilty simpliciter, feeling that one is essentially guilty or burdensome - that one could not have been otherwise or is to blame for one's depression - negative self-narrative, over-active rumination and repetitive thinking which reduces one's ability to concentrate).

- Disturbances to individual's sense of agency or free will (acts seem not only difficult but impossible, an impossibility which affects the way the world is experienced when one is depressed).

\footnotetext{
${ }^{7}$ For causal views of mental disorder, see (Beebee and Sabbarton-Leary [2010]; Kendler et al. [2010]; Murphy [2014]).

8 Prima facie, the disturbances to one's mental life that are associated with MDD for example, don't seem to mere causal effects of the illness in the same way that the abdominal pain commonly associated with having appendicitis does.

9 See (Radden and Varga [2013]) for an epistemological defence of the reliability of first-person reports of depression which this discussion relies upon.
} 
- Changes to the subject's experience of time (time seems to 'slow down' or 'stop' when one is depressed, a change which is attributable to changes in the overall structure of temporal experience as well as changes to the rate of 'temporal flow', the future appears 'closed' to depressed agents, - a feature which is connected to the way in which individuals find things salient or significant).

- Changes to the structure of interpersonal experience (feelings of estrangement, changes to how a person relates to another as a person, loss of interpersonal connection, being cut off from the world and others).

Ratcliffe's discussion of these phenomenological changes is extensive. The main idea which I wish to highlight here is the central claim or overarching view of depression which Ratcliffe claims is supported by a wide range of reports of depression. This is the idea that while MDD seems to reliably track the range of specific content-related changes just listed, individuals who suffer from depression frequently report that the experience of depression cannot be reduced to these types of changes ${ }^{10}$. That is, the report that any attempt to explain or describe depression solely in these terms misses something crucial; a feature of depression which is often connected to the assertion that depression is, even among mental disorders, particularly elusive ([2015] p.2; Ratcliffe et al. [2013]) ${ }^{11}$. This pushes Ratcliffe to endorse a view on which depression, rather than involving a conjunctive set of changes to one's experience, involves a change to something more general and fundamental to experience. On his view, first-person descriptions of MDD support a view of depression as involving a radical and allencompassing change to the total structure or fabric of a subjects' experience, one in which the robust subjective changes listed above take place within, or are to be made sense in terms of:

"Sufferers consistently indicate that depression is qualitatively different from what many of us regard as 'everyday' experience. The depressed person finds herself in a different 'world', in an isolated, alien realm that is cut off from the consensus reality where people have more mundane experiences of feeling 'more $x$ ' or 'less $y$ ' than usual...Depression instead involves a disturbance of something that is fundamental to our lives, something that goes unnoticed when intact. What is eroded or lost is a 'sense' or 'feeling' of being comfortably immersed in the world ([2015] p.10, emphasis added).

According to Ratcliffe, this shift explains a number of salient features about depression as a phenomenon. For example, it is argued that this explains why individuals diagnosed with MDD often report that depression is elusive and hard to convey to others who have not experienced it. It is because depression is not reducible to a conjunction of particular changes to the content of one's

${ }^{10}$ Where this is not to be understood as a general claim about phenomenal experiences but a specific claim about the experience associated with depression.

11 Many conscious experiences are difficult to describe precisely. This is particularly true of what we might call 'extreme' experiences which plausibly reflect individual differences in mental traits of organisms (Machery [2017]) the experiences associated with the absence or extremely vivid forms of imagination are a good example here (Zeman et al. [2020]). The claim Ratcliffe is highlighting with respect to depression is stronger. The idea is that individuals with depression lack an appropriate vocabulary or frame of reference with which to describe their experience; a claim exemplified in the reports that being depressed is to be "cut off from the range of normal experience of feeling more $\mathrm{x}$ or less y' and is closer to being 'beyond description', rather than merely being difficult to convey ([2015]; pp. 2-3). 
experience (having a lower mood than usual, feeling more guilty than I did before) but involves a change to something more fundamental (but elusive), that it is difficult to convey to others what it is like to be depressed ([2015] p.2; p.158).

With this in mind, it is easy to appreciate how Ratcliffe's phenomenological analysis challenges the sufficiency of the two views of depression which emerge from the clinical characterisation of MDD. If we take Ratcliffe's claim about the mental changes which occur when one is depressed at face value, for example, current descriptivist views appear to be inadequate or incomplete because they make no reference to this change, nor many of the important aspects of it ${ }^{12}$. This is clinically problematic insofar as it implies that the current DSM-5 category MDD may include a variety of other predicaments which are distinguishable from depression (and thus should not be identified with it) ${ }^{13}$. Similarly, and derivatively, Ratcliffe's analysis raises problems for existing causal views which equate depression with a particular causal explanation for MDD's diagnostic symptoms. Here, the thought is that the causal view is doubly inaccurate because it not only overlooks the fact that there are subjective features essential to MDD (viz. the fundamental 'shift' described above) but also in virtue of the fact that it asserts that depression is to be identified with the causal explanation for these coarse-grained symptoms. The causal view thus inherits the problems of ontological descriptivism and gains a few problems of its own - it searches for a causal explanation of a phenomenon in the wrong place because it has an incorrect, or ill-defined explanandum. In both cases, the central complaint here is the same: the phenomenological analysis of depression offered by Ratcliffe provides us with reason to think that these accounts miss out on or fail to acknowledge a crucial level of explanation necessary for understanding and diagnosing depression, namely, one which makes reference to the fundamental and structural features of an individual's conscious experience ${ }^{14}$.

For Ratcliffe, these first-person reports of depression motivate a phenomenological theory of depression on which depression centrally involves an 'existential shift' to one's experience. Depression, on this view, involves a change in 'existential feeling' which corresponds to the sense of 'world' examined by phenomenologists such as Husserl, Heidegger and Mearlau-Ponty ${ }^{15}$. Ratcliffe's phenomenological theory here is connected to his general endorsement of a methodological

\footnotetext{
12 That is, even to the extent that they do attempt to account for these changes (in a 'depressed mood' for example) Ratcliffe's phenomenological analysis highlights a further limitation with this account viz. that these descriptions are provided at the wrong level of explanation - that is, are cashed in in terms of changes to particular contents of an individual's experience. If the phenomenological claim which emerges from (Ratcliffe [2015]) is correct, then any criteria described solely at this level will leave out something crucial.

13 This seems to be Ratcliffe's point: 'a central theme of this book is that diagnostic categories such as Major Depressive Disorder are insufficiently discriminating and accommodate a variety of predicaments' ([2015] p.75).

${ }^{14}$ For recent discussion of multi-levelled causal explanations in psychiatry see (Kendler and Gyngell [2020]; Cooper [2020]).

${ }^{15}$ See Ratcliffe (2015) Chapter 2 for a comprehensive statement of this idea, which is intimately connected to the kinds of possibility one can experience. Fernandez ([2014]) and Saarinen [(2018]) offer further elaboration of this phenomenological theme.
} 
'phenomenological stance' with respect to depression, “through which one comes to appreciate that certain questions cannot be satisfactorily addressed from the standpoint(s) of empirical science" ([2015] p.21). In my view, Ratcliffe's specific phenomenological theory about depression can and should be separated from the general observation that his work highlights viz. that depression involves a profound change or shift to the structure of one's experience. This latter claim ought to be regarded as an important source of data for psychiatric research on the nature of depression. One can, I think, accept both that first person reports are useful, that they point to the fact that something is missing in current attempts to characterise depression, without thinking that this is or must be something which is not amenable to empirical investigation.

From an empirical perspective however, the claim that depression involves a profound change to the structure of one's experience (that is, a change to something 'fundamental' which 'goes unnoticed when intact' ([2015] p.2) - particularly in light of the phenomenological theories of depression associated with it - appears at first glance to be something mysterious. What is this fundamental change that occurs, and how could this possibly be understood and studied in scientific terms? In the following section I outline an answer to this question, an answer which has only become available recently due to advances in consciousness science. This is the thesis that depression involves a change to one's global state of consciousness ${ }^{16}$.

\section{What is a Global State of Consciousness?}

Changes to the content one experiences are not exhaustive of the changes to one's experience that are possible, or that even occur regularly. This fact becomes salient when we reflect on what happens to our experience when we fall asleep each day and enter a dream, or when we emerge from a dreamless sleep into waking consciousness. In these common and everyday cases, one's experience undergoes a distinctive change - the experiences of dreaming and wakefulness being often very different which does not, prima facie, seem to be describable solely in terms of specific changes to the content

\footnotetext{
16 The preceding discussion is suggestive of two distinct readings of Ratcliffe's position which are worth noting here. These arise from an ambiguity in the assertion that "certain questions cannot be satisfactorily addressed from the standpoint(s) of empirical science" ([2015] p.21). On an initial reading, "cannot be satisfactorily addressed" can be taken to imply that there are certain questions one can ask about depression which are not amenable to empirical investigation in general or at all, given the nature of empirical enquiry. This sort of claim is in tension with the central proposal put forward in this paper, and is thus a claim I reject. However, there is a weaker reading of Ratcliffe's assertion here which complements the positive hypothesis I develop, on which Ratcliffe commits only to the idea that there are certain questions about depression that cannot be addressed satisfactorily by empirical science alone or to begin with. Here, for example, one might view the adoption of the phenomenological stance as a necessary but initial step in a longer process of naturalisation; one which seeks to subsequently integrate phenomenological observations and insights into an empirical account of depression (for example, by identifying the proximate neurobiological causes of these phenomenological changes, or by translating them into a terminology which is amenable to empirical investigation). Thank you to an anonymous referee for prompting me to detail this alternative reading of Ratcliffe's position.
} 
one experiences ${ }^{17}$. In consciousness science and the philosophy of mind, such changes are referred to as a subject entering into a different global state of consciousness, paradigmatic examples of which include wakefulness, lucid and non-lucid dreaming, drug-induced psychedelic states and postcomatose disorders such as the minimally conscious (MCS) and vegetative states (VS) ${ }^{18}$.

As a central theoretical construct in consciousness science, a recent body of work has sought to understand and conceptualise what global states of consciousness consist in, such as to account for what distinguishes different global states (for example, dreaming and the psychedelic state) from each other ${ }^{19}$. While research on this is still on-going, there is an emerging and promising conceptual framework for understanding global states of consciousness which provides a firm foundation for my proposal. This is the claim that global states of consciousness are states of systems which regulate the range of cognitive, attentional and content-related capacities that are online for a subject. I unpack this below 20 .

A view on which global states of consciousness are states of multi-dimensional capacitation takes as its starting point the rejection of a traditional conception of global states which understands these in terms of 'conscious levels'. On this traditional view, born out of the clinical literature on postcomatose disorders, consciousness (in the global sense) is a gradeable property which admits of a linear ordering from states which are 'least conscious' to those which 'most conscious' [Figure 1]. For example, this view characterises Minimally Conscious State patients as having a higher level of consciousness than Vegetative State patients do, and so on with respect to the global states associated with dreaming, wakefulness and psychedelics ${ }^{21}$.

17 See for example Windt's ([2015]) influential argument that dreaming cannot be characterised in the standard psychological terms used to characterise changes in wakeful consciousness. This serves to distinguish global states from other sorts of mental phenomena which occur within a particular global state such as emotions, thoughts or moods.

18 These are also sometimes called "background states" (Chalmers,[2000]), "levels" (Boly et al. [2013]; Laureys, [2005]), "modes" (Bayne \& Hohwy, [2016]), "global modes" (Fortier-Davy \& Millière [2020]), and "aspects" (Klein, [2017]) of consciousness (McKilliam [2020]).

19 Within consciousness science, the concept of a global state is typically contrasted with the notion of 'local states' of consciousness or 'conscious contents' (occurrent experiences such as perceptual experiences of various kinds, imaginative and affective experiences, experiences of agency, and occurrent thoughts) which together form the basis of the Neural Correlates of Consciousness research programme (Koch et al. [2016]; Boly et al. [2017], Klein et al. [2020]). In addition, the notion of global states has been gaining prominence as a result of work on the nature and constitution of specific global states such as dreaming and wakefulness. See (Windt [2015]; Voss and Hobson [2014]; Crowther [2018], Soteriou [2020]) for a short, non comprehensive list of such work.

${ }^{20}$ The account I will sketch here draws mainly on ideas found in (Bayne et al. [2016]); Bayne and Carter [2018], Crowther [2018], Fortier-Davy and Milliere [2020], Mckilliam [2020].

21 See (Laureys [2005]; Koch et al. [2016]; Boly et al. [2013]; Overgaard and Overgaard [2010]. 


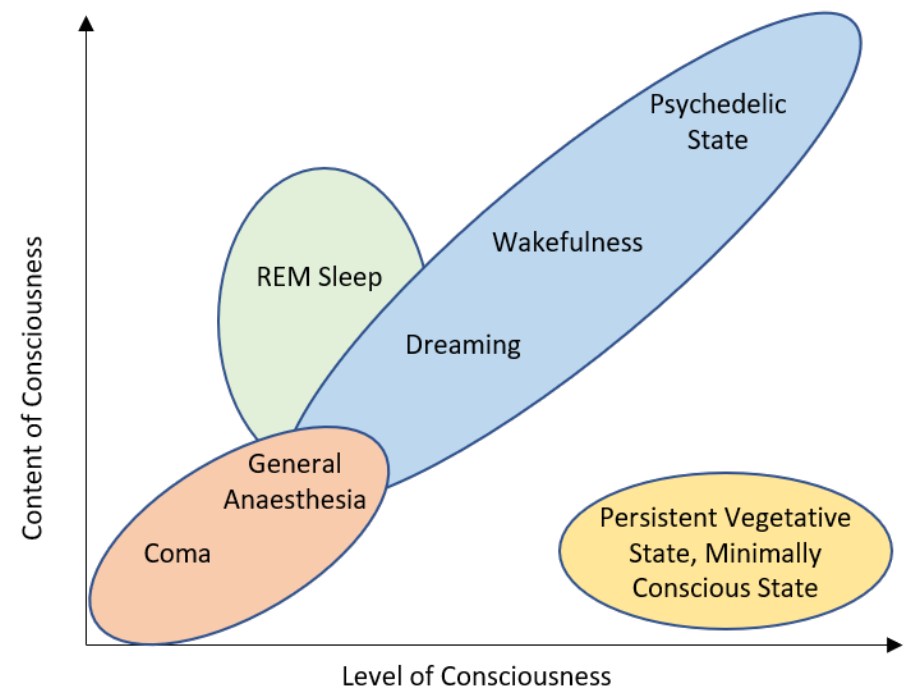

Figure 1: The Levelled Conception of Global States (adapted from (Bayne et al. [2016] p.406)

This levels based view has been objected to on several grounds. One central concern, found in (Bayne et al. [2016]), comes in the observation that while there may be an intuitive sense in which some global states of consciousness are 'lower' than others - for example, when comparing the PVS with wakeful consciousness - further conceptual and empirical reflection reveals that global states of consciousness in fact differ from each other in multiple respects and are not measurable along a single dimension[Figure 2] $]^{22}$. For example, while there is an intuitive sense that the psychedelic state one enters after having taken psychedelic drugs is a 'higher' state of consciousness than the wakeful state we typically occupy, on closer inspection we find that a strict ordering is not possible here: there will be some aspects in which the psychedelic state is improved or enhanced relative to wakefulness - such as the vividness and intensity of the sensory contents one perceives - but many aspects of a subject's experience are impaired in such states - for example, an agent's decision making and attentional capacities (Bayne and Carter [2018]). Similarly, some aspects of the psychedelic state will be neither enhanced nor impaired but different or novel in a way which is not strictly comparable to wakefulness (for example, the experiences of ego dissolution frequently reported at high dosages of psychedelics) ${ }^{23}$. Thus, while a partial ordering may be possible, the linear ranking implicated in a levels-based conception of global states appears unfeasible.

\footnotetext{
22 Bayne et al. ([2016], p.407) also argue against the levelled view of conceptual grounds, on the basis that our concept of consciousness does not admit of degrees.

${ }^{23}$ In this sense, Bayne et al. ([2016] p.409) write that global states are similar to economies. Just like one cannot compare two economies on a single dimension, but along several different dimensions, they argue that global states of consciousness similarly resist comparison in absolute terms.
} 


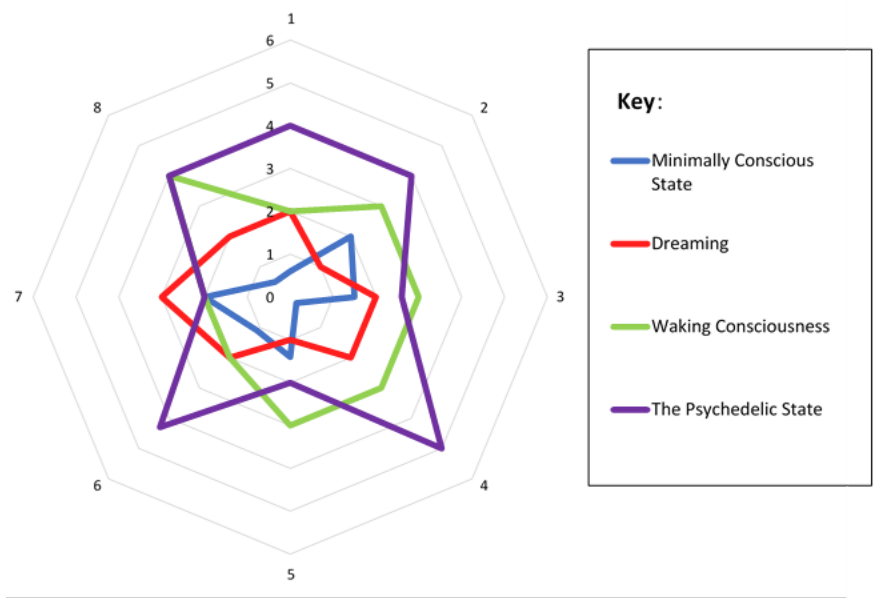

Figure 2: The Multi-Dimensional View of Global States (adapted from (Bayne et al. [2016]). This generalises to all global states of consciousness. As in Bayne et al., the number of dimensions represented here, as well as the placement of particular global states along them, is only intended to be illustrative and does not commit to a claim about the actual or ideal number of dimensions of the global state space. This remains an open empirical question. For now, the reader can view the eight dimensions depicted here as corresponding to a range of dimensions taken from the four dimension families described below.

In light of this, Bayne et al. ([2016]) propose an alternative conceptual framework for global states of consciousness according to which global states of consciousness are multi-dimensional constructs measurable across several different dimensions. The search for a comprehensive list of the dimensions which frame global states of consciousness is still on-going. However, recent literature suggests that there are nonetheless several families of dimensions which characterise the global state space. These dimensions, which are drawn from a contrastive examination of different global states of consciousness, are listed below:

\section{Dimensions of the Global State Space:}

(i) Sensory content (corresponding to the intensity and volume of contents experienced (Bayne et al. [2016]) and the quality of conscious contents (Fazekas and Overgaard [2016]).

(ii) Cognitive content (corresponding to mental control and manipulation, attentional capacities and concentration, (enhanced or impaired) capacity to formulate and distinguish novel thoughts (Bayne and Carter [2018]).

(iii) Subjective dimensions relating to the subject's experience of unity, self and time (corresponding to the experience of time as stopping or slowing, an openness to past and present, altered experiences of self, the boundary between self and environment, experiences of 'egodissolution' and experiences of disembodiment (Bayne and Carter [2018]). 
(iv) Functional dimensions (corresponding to the extent to which information can be globally broadcast, both in terms of the ability to which conscious contents can be broadcast, and the range and number of consumer systems it can be broadcast to (Bayne et al. [2016]; McKilliam [2020])24.

This work raises an obvious question regarding how these dimensions ought to be understood and characterised precisely. According to a recent proposal, broadly continuous with Bayne et al.'s multidimensional framework, the dimensions which frame the global state space ought to be analysed in terms of capacities which are currently online for a subject (Crowther [2018]; Soteriou [2017]; [2020]; Fortier-Davy and Millière [2020]; McKilliam [2020]). One way of motivating this claim, drawn from the philosophy of mind, is via an examination of the nature of the dreaming state - in particular, the claim that when one is dreaming when one is in a general state of incapacitation (Crowther [2018]). This reflects the general idea that there are capacities that individuals can only exercise while awake which the individual is unable to exercise whilst asleep and dreaming; when the individual is asleep, these capacities remain but are 'fettered' in some way (Soteriou [2020])25. The 'capacities account' outlined by McKilliam ([2020]) generalises this idea to all global states of consciousness. This gives the proposal in this paper clear positive content:

Global states of consciousness: states of creatures (systems) that regulate (i) the range and quality of conscious contents the creature is capable of experiencing while in that state, (ii) the range of cognitive systems into which those contents can be mobilised while in that state, and (iii) the range of attentional capacities the creature has while in that state. (Mckilliam [2020] p.14) ${ }^{26}$.

According to this capacitation account then, global states of consciousness are characterised by a profound shift in a content of an subject's experience which is constitutively connected to the fact that when one's global state of consciousness changes, the range of content, cognitive and attentional capacities that are online for the subject changes. Thus, what is central to occupying, for example, a psychedelic state as opposed to a state of wakefulness is a simultaneous structural change to

\footnotetext{
${ }^{24}$ The reader who is familiar with consciousness science will have recognised that as listing some of these features as dimensions, as opposed to constitutive features of consciousness, the authors' claims seem to be in tension with some major theories of consciousness. For discussion see (Bayne and Carter [2018]).

25 One might interpret this as the claim that when an individual is asleep a range of capacities she is able to exercise while awake are masked (Fara [2008]). In this sense, global states are markedly distinct from other mental phenomena such as moods which (while they may dispose creatures to experience certain emotional content (Textor and Grzankowski unpublished)) do not regulate which capacities for certain experiences are online for a creature.

26 The notion of regulation utilised by McKilliam is ambiguous between a strong view of global states as causally acting or regulating these capacities in order to keep them on or offline (one might think that sleep is a good example of a state of this kind) and a weaker view on which one views global states as a cluster of on/offline capacities which regularly co-occur in something like a homeostatic property cluster sense (Boyd [1999]). The weaker claim is all that I commit to here.
} 
perceptual, cognitive, self and temporal-related experiences which is reflected in a profound and holistic change to one's conscious experience (Bayne and Carter [2018])27.

Replacement of the levelled based view of global states with the multi-dimensional account has opened up numerous exciting research avenues within consciousness science. One such possibility, which has not yet been discussed, is that the multi-dimensional view, in contrast to the previous levelled conception, prompts us to ask what other psychological phenomena may fall under - or be best explained in terms of - a change to one's global state of consciousness. I explore this in the next section, where I develop the hypothesis that depression is one such phenomenon which is apt for explanation and elucidation in these terms.

\section{The Depressive State of Consciousness}

In Section 2 I argued that an application of Ratcliffe's analysis, which seeks to challenge existing accounts of depression on the basis of their inability to account for the phenomenology of depression, faces a significant challenge with regard to reconciling this idea with a naturalistic approach to psychiatry. This paper's central claim is that the recent advances in consciousness science just described provide a way of meeting this challenge. Here, I'll develop the hypothesis that when an individual is depressed, she departs from a state of wakefulness to a distinctive depressive state of consciousness, a change which is reflected in an experience of an 'existential shift' as described by Ratcliffe. As stated, the aim here is to provide conceptual motivation for this hypothesis via the claim that this can account for the phenomenology of depression. The real test will come in its empirical applicability, which must come later ${ }^{28}$.

On the view I am proposing, the changes an individual undergoes when she becomes depressed resemble - and are changes of the same kind as - the changes to her experience that occur when she departs from waking consciousness and starts dreaming, or when she enters a psychedelic state. In the context of the phenomenological features of depression outlined in Section 2, the central motivation for this claim is twofold. First, an understanding of depression as a global state of consciousness captures the central phenomenological claims highlighted by Ratcliffe, namely, that depression, while

\footnotetext{
${ }^{27}$ A crucial question for the capacitation account just sketched which has yet to receive significant attention concerns the relation between the capacities which characterise global states and the specific states e.g. 'wakefulness' 'dreaming' themselves. On the view just sketched, the correct relation between these is a reductive one: on this view, to be in a global state of consciousness such as dreaming just is to realise a set of values which correspond to a range of consciousness-related capacities. However this view may be objected to. One line of resistance is the observation that such an account necessarily fails to recognise that the capacity related dimensions which frame the multi-dimensional space interact in ways specific to a given global state of consciousness. Relatedly, one might reject this view on the basis that such an account does not allow for the possibility of a non-reductive view on which a given state of consciousness ('wakeful consciousness') plays explanatory role(s) over and above the explanatory roles of online capacities (for example, with respect to knowledge (Soteriou [2020]; O'Shaunessey [2002])). While I am sympathetic to a non-reductive view, I leave this issue open here as the hypothesis I offer is not dependent upon its acceptance.
}

28 I include a brief suggestion as to how this might be implemented in Section 6.1 . 
often characterised by numerous specific changes to the content of experience (relating to time, mental agency and so on), resists explanation in these terms in a way which distinguishes it from other sorts of experiences. That is, that depression appears - to those experiencing it - to involve a disturbance or shift to a more fundamental aspect of experience. The hypothesis about depression under consideration here offers an explanation as to why depression has these features. The 'shift' it denotes marks a change from one global state of consciousness to another (an elusive process which one is often not explicitly aware of) while the former claim is captured by the fact that global states also distinctively resist analysis at the level of conscious content. In both depression and global states of consciousness, there is a level of explanation - that is, one at the level of global changes to experience - required to account for the phenomenon; a level of explanation which disappears, or is left out, when one attempts to describe the phenomenon wholly at the level of conscious content.

The second line of motivation reflects the multi-dimensional capacitation account of global states outlined above. This is the striking observation that many of the changes to experience reported by individuals diagnosed with MDD - those documented extensively by Ratcliffe - are experiential changes of the same kind as those reported by Bayne and Carter ([2018]) with respect to the psychedelic state. In both cases, individuals report robust phenomenological changes or alterations to their experiences of time, their sense of self, bodily experience, mental agency, concentration and attention (Ratcliffe [2015]; Bayne and Carter [2018]). In both cases, these changes do not tend to happen disparately but appear, from the reports, to come into effect all at once. The hypothesis here is, again, well placed to make sense of and explain the phenomenological similarities here. The thought is that both of these phenomena involve a change to an individual's global state of consciousness, and changes to one's global states of consciousness are just the sorts of thing which involve simultaneous experiential changes along these dimensions. In addition, and perhaps yet more telling, are the numerous observations that for many individuals who are depressed it is the capacity for certain sorts of experiences - what Ratcliffe refers to as 'existential' forms of experience, like the capacity to hope - and not merely the degradation or enhancement of ordinary forms of experience that go missing 29 . Here again, the hypothesis that depression involves a change to one's global state is well placed to explain this feature, via the assertion that global states are precisely the sort of thing which regulate the range of mental and content related capacities which are online for a creature.

The general hypothesis that depression involves an altered state of consciousness can be fleshed out by drawing on the multi-dimensional framework outlined in the previous section. This provides the beginnings of a more specific interpretation of the thesis that depression involves a change to one's global state of consciousness according to which, when one is depressed one enters a state that regulates changes to the range and quality of conscious contents one is capable of experiencing, the

${ }^{29}$ Indeed, this phenomenological distinction is one of the central themes of the book. See, for example, ([2015] Chapter 4 'Loss of Hope'). 
range of cognitive systems into which those contents can be mobilised, and the range of attentional capacities one has. But what are these capacities more specifically? The material in Section 3 allows for a more detailed characterisation of the depressive state as follows ${ }^{30}$ :

Dimensions of the Depressive State: depression is a state of consciousness whose key capacity related dimensions include: (1) dimensions relating to bodily experience (feelings of fatigue, tiredness, lethargy, heaviness, sickness or nausea, loss of appetite, numbness and sense of agency) (2) dimensions relating to cognition (impairments with respect to mental control, impairments to an agents attentional capacities and concentration, the capacity to experience hopeful thoughts, an increased capacity for a range of guilt related thoughts, and impairments to self-narrative) and (3) dimensions relating to the subject's experience time (changes in the overall structure of temporal experience, changes to the rate of 'temporal flow', the future appears 'closed' to depressed subjects) (4) dimensions relating to the subjects experience of others (feelings of estrangement, changes to how a person relates to another as a person, loss of interpersonal connection, being cut off from the world and others).

Like existing characterisations of specific global states such as the characterisation of the psychedelic state offered in (Bayne and Carter [2018]), this multi-dimensional characterisation of depression is provided with the expectation that this will require further development and revision as further work on depression and global states of consciousness emerges. Nonetheless, it is still informative enough to do explanatory work, which I return to in the next section. Before I do so however, further clarification of my hypothesis is required. In particular, more needs to be said in order to render this proposal consistent with a number of claims which are obviously true with respect to depression. This is that when one is depressed one is (i) still awake, (ii) one retains the capacity to dream, (iii) that one may return to an ordinary state of wakeful consciousness for intermittent periods while depressed, and finally (iv), that we should leave open the possibility that there may be different sub-kinds of depression, yet to be distinguished, which are lumped together on the current characterisation I have provided.

There is a straightforward way of accounting for (i) which readers who are familiar with the literature on post-comatose disorders will recognise. The strategy here appeals to the distinction between a subject's being awake (e.g. a state associated with various levels of arousal) and that same individual being in a state of wakeful consciousness ${ }^{31}$. The sense in which depression constitutes a departure from waking consciousness would then, on this view, resemble the departure from waking consciousness that occurs in the standard post-comatose disorders as well as in individuals who are in psychedelic states. This is straightforward. The points implicated in (ii-iv) however require further conceptual scaffolding. I propose that we can account for these features of depression if we take a step back from

\footnotetext{
30 This is taken to be analogous to the broad characterisation of the psychedelic state recently offered by Bayne and Carter ([2018]).

31 Soteriou [2020]; Owen [2009]; Bayne and Shea [2010]; Laureys [2005].
} 
consideration of individual depressive episodes and account for their occurrence within a broader understanding of 'depression' as an extended temporal process [Figure 3].

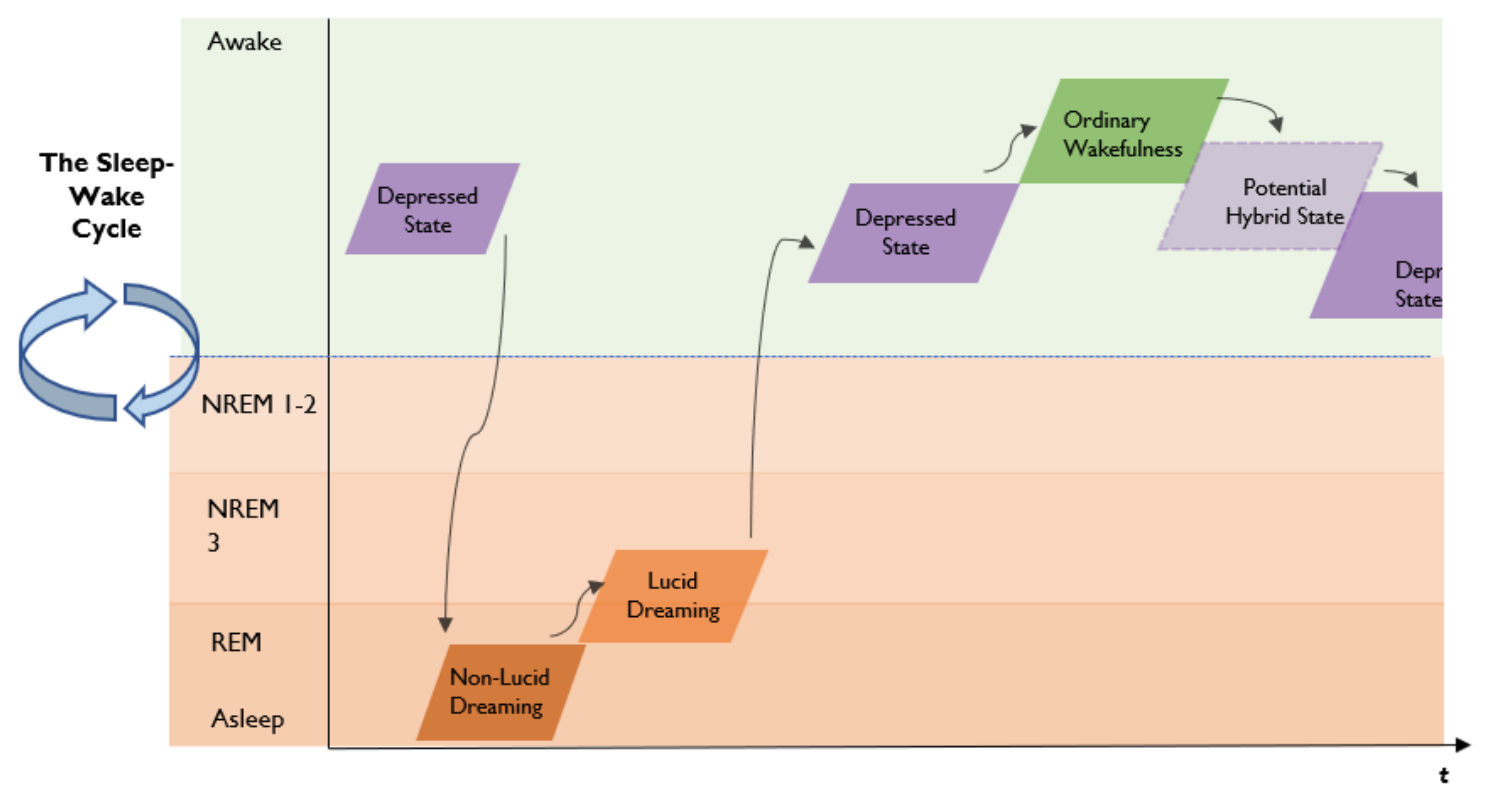

Figure 3: Depression as a temporal process with the depressive state as temporal parts. This is an over simplistic representation of the actual process in several respects. For example, the changes between entering one state of consciousness from another, particularly in the wakeful-dreaming case are often non-instantaneous, and the recourse back to occupying a state of non-pathological wakefulness is likely to occur for periods of days or months rather than hours. Similarly, the placement of dreaming in relation to sleep stages in overly simplistic insofar as REM sleep and dreaming are now understood to be doubly dissociable.

On the view I have in mind, an individual who is depressed is so characterised in virtue of the fact that this individual frequently (but not invariantly) occupies a depressive state of consciousness where one would typically occupy a state of ordinary wakeful consciousness. Thus characterised, a diagnosis of depression - and the hypothesis that depression involves a departure from wakefulness to a distinctive depressive state of consciousness - is consistent with that same individual sometimes occupying healthy wakeful states of consciousness for periods of time (as per the common observation that depressive episodes often obtain intermittently and not continuously) ${ }^{32}$. This is also consistent with that same individual frequently reporting experience of dreams ${ }^{33}$. Here again, the idea would be the same: if one understands depression as a broader temporal process it is the frequent recourse back from dreaming and other sleep states to the depressive (non wakeful) state that is important for capturing the sense in which that individual is depressed. Finally, this view also accommodates the

32 This periodicity is a common feature of diseases. A good example in the mental realm is in individuals diagnosed with Tinnitus. Here, an individual can warrant a diagnosis of Tinnitus despite not having the central feature of this disorder continuously, either in virtue of the fact that the individual only 'hears' the distinct sounds intermittently (in the case of 'Intermittent Tinnitus') or in virtue of the fact that these are not perceived whilst the individual is dreaming (Henry et al. [2014]).

$33 \mathrm{~A}$ further explanatory virtue of this hypothesis is that it is well placed to account for the strong empirical connections made between sleep, dreams and depression (Thase [2006]; McNamara et al. [2010]; Aviram and Soffer-Dudek [2018]). 
possibility that depression could admit of various sub-kinds which may correspond to different levels of severity. On the view proposed here, these sub-kinds would themselves amount to distinct global states of consciousness which would constitute hybrid states between the depressive state and wakefulness ${ }^{34}$. This allows for the further possibility and that an individual may - over the course of their depression - occupy several of these states over a long period of time ${ }^{35}$.

In closing, I want to consider where this leaves us vis-a-vis the view of depression endorsed by ontological descriptivists and causalists. The hypothesis just outlined is, I think, consistent with a revised version of either of these views. This follows from the observation that the claim that I am making here is one of a necessary condition on a diagnosis of depression. This can be accommodated both in terms of a descriptivist view which acknowledges the depressive state (or aspects of it) as central diagnostic symptoms of depression, and with a biomedical, causal view if the depressive state is taken as a central explanandum of the constitutive explanation provided. A distinctive global state of consciousness will certainly have a constitutive mechanistic explanation, in accordance with a biomedical or biopsychosocial view (more on this below) which renders it compatible with a causal account . There is however a third view of depression to which this hypothesis gives rise, which I wish to mention briefly in closing, without endorsing. This is one on which depression just is the sort of extended temporal process involving depressive states sketched above. On this view, which would constitute a sort of middle ground between descriptivism and causalism about depression, depression qua depressive states is both an explanans and explanandum. That is, the depressive state would itself have a constitutive mechanistic causal explanation and work to explain a number of causally downwind effects or diagnostic symptoms of depression (such as loss of appetite, or sleep disturbances). Yet, crucially, on this view, it would be inappropriate to identify depression with either of these.

\section{Empirical Evidence and Application}

The argument so far has attempted to show that there is a viable analysis of the 'phenomenological shift' evidenced in first-person reports of depression which is explicable in naturalistic terms. This view has thus far been motivated by phenomenological considerations: it is attractive, I have argued, because it alone has the resources to explain why depression feels - from the inside - the distinctive way it does. This should prompt philosophers and psychiatrists to take it seriously. Depression, however, has other distinctive features which reveal themselves in the empirical literature on MDD. These are often expressed in the form of questions or puzzles: Why is there currently no single

\footnotetext{
34 This would thus amount to a waking analog of lucid dreaming which has been proposed as hybrid state of consciousness between wakefulness and dreaming (Valat and Ruby [2021]; Voss et al. [2009]).

35 Ratcliffe ([2015]) suggests something like this hypothesis and it is implicated in much of the clinical literature on depression (Otte et al. [2016]). One might connect this idea to the observation that MDD is frequently co-morbid with other disorders such as anxiety (see Richards and O'Hara [2014], along with the 'specifiers' of MDD listed in the DSM-5).
} 
mechanistic or neurological explanation for depression which can explain various aspects of the disorder? How are we to best intervene and treat depression, when such a neurobiological explanation is not forthcoming? In this last section, I will outline two important empirical implications of this hypothesis which shed light on these questions. The aim here is not to offer anything like the final word on these, but rather to point to several exciting lines of research which can be taken up in the future.

\subsection{Global State and MDD Research: A Reciprocal Relationship}

A central line of criticism posed against MDD as a diagnostic category is grounded in the fact that despite significant advances in the understanding of the neurology of many mental disorders, there is currently no single neural mechanism identified which can explain all aspects of depression ${ }^{36}$. One explanation for this is that there is no unified kind here at all: while at first glance MDD appears phenomenologically unified, upon empirical investigation, we find that there is no neurological unity to these reported cases of depression. It thus may follow that we ought to eliminate the category from our scientific discourse, and replace it with a series of more fine-grained categories which correspond to the neurologically unified mechanisms we do find ${ }^{37}$.

This eventuality should not be ruled out. However, if the hypothesis I am proposing here is plausible, such a conclusion may be premature. Here, the thought is that the hypothesis that depression involves a distinctive depressive state of consciousness may help explain why a mechanistic explanation for depression has not yet been forthcoming. This is due to the fact that there is currently no established account of the neural mechanism(s) underlying global states of consciousness ${ }^{38}$. This is to say, on the view that depression involves a change to one's global state of consciousness, the fact that a mechanistic explanation has not been found for depression is unsurprising, for this would amount to a mechanistic explanation for a global state of consciousness.

Thus interpreted, the hypothesis here interestingly offers us reason to be cautiously optimistic about the discovery of a mechanistic account of depression within the next few decades. In particular, the hypothesis here opens up the possibility that, as the work in consciousness science continues to progress, this can be used to inform and motivate new lines of research into the mechanistic basis of depression. In time, this work may allow for the production and implementation of empirical and

\footnotetext{
36 However, a number of common biomarkers have been identified (Otte et al. [2016]). Time constraints permit me from discussing these here. For a recent meta-analysis of this research which challenges the predictive success of these biological markers see (Kennis et al. [2020]).

37 This is the line of thought often driving discussion of the $\mathrm{RDoC}$ as an organising framework for psychiatric research (Insel [2014]).

38 Although this research is well underway. See (Howhy and Bayne [2016] and Koch el al. [2016]) for overviews of current research and main predictions made NCC research, and (Revonsuo [2006] and Owen and Guta [2019]) for arguments that this ought to be understood in terms of neural mechanisms.
} 
objective tests for depression in patients using fMRI and other neuroimaging techniques employed for the purposes of detecting changes to global states of consciousness. While much of this will depend on future developments within the NCC research programme, work along these lines may already be possible. I have in mind here the potential application of what is known as the "perturbational complexity index (PCI)', which utilises transcranial magnetic stimulation (TMS) to engage distributed interactions in the brain and to measure their algorithmic complexity (Casali et al. [2013]; Sitt et al. [2014]; Schartner et al. [2015]; Hudetz et al. [2016]). PCI has been shown to reliably discriminate global states of consciousness in individuals during wakefulness, sleep, and anaesthesia, as well as in patients in the MCS, with high PCI values associated with wakefulness and psychedelics (Schartner et al. [2017]) and low values in impaired conscious states like the clinically diagnosed VS and MCS patients. Accepting that the PCI can be used as a correlate or marker of (as opposed to constitutive of) an individual's global state of consciousness opens up a potential empirical line of research which can begin to shed light on the viability of the hypothesis outlined in this paper. That is, accepting the correlational utility of the PCI, the hypothesis in this paper suggests that individuals diagnosed with MDD would, as a whole, exhibit statistically significant different PCI values relative to healthy controls subjects ${ }^{39}$. Such a study would thus be clinically worthwhile.

On the other hand, the hypothesis defended in this paper also has general applicability and utility for furthering research into the nature and neural bases of global states of consciousness. This can be done both at a personal and sub-personal level. On the subjective level, for example, the characterisation of the depressive state outlined in the previous section provides a new resource for identifying the subjective dimensions which characterise the general global state space (Bayne et al. [2016]). While this works to reinforce many of the dimensions outlined by Bayne et al. and Bayne and Carter, the dimensions drawn from Ratcliffe's phenomenological analysis also highlight new families of dimensions - particularly, those relating to bodily experience and intersubjectivity - which have not yet been properly considered in the on-going research on global states. The hypothesis here encourages the investigation of these dimensions in the contexts of other global states of consciousness. In addition, on a sub-personal or neurological level, the hypothesis here encourages the application of the various biomarkers of depression (Otte et al. [2016]) to NCC research, in order to facilitate new lines of research on the neural basis of global states which may currently be overlooked.

\subsection{Psychedelic Psychiatry}

The search for a mechanistic basis for depression is important not only for the purpose of vindicating MDD as a diagnostic category, but for the purposes of facilitating improved treatment options for depression. Currently, two main treatment options for MDD are available, including psychotherapy

\footnotetext{
39 While there is early evidence that depression is associated with higher levels of brain complexity (Li et al. [2008]), a
} comprehensive study using the PCI is lacking. 
and pharmacotherapy ${ }^{40}$. As is well known, both psychotherapy and pharmacotherapy have limited efficacy as treatment options. Both have been shown, for example, to be effective in only around $50 \%$ of cases, a statistic which continues to spur the need for development of new therapeutic interventions ${ }^{41}$. Within this vein, a promising avenue of research examines the therapeutic potential of psychedelics (such as Ketamine, Mescaline and Psilocybin, the active ingredient in magic mushrooms) as a treatment for depression (Rucker et al. [2018]; Nutt [2019]; Nutt et al. [2020]) ${ }^{42}$. While research on psychedelic psychiatry is still in its early days, initial results have been promising. For example, four separate trials have reported improvements in depressive symptoms after psilocybin-assisted psychotherapy (Griffiths et al. [2016]; Ross et al. [2016]; Grob et al. [2011]; Carhart- Harris et al. [2016]), including one in which 'treatment-resistant depression' was the primary criterion for inclusion (Carhart- Harris et al. [2016]) ${ }^{43}$. As a result, major research centres have now been set up at Imperial College London and John Hopkins University to study the therapeutic potential of psychedelics for depression and related disorders.

A central question for this research, and one to which answers are currently speculative, concerns the explanatory rationale for psychedelic psychiatry. The key question is as follows: what explains the current positive results of psychedelic therapy, and what ought to motivate the funding of further research in this field? (Letheby, [forthcoming]). Current answers to this question offered by leading researchers in this area, locate this rationale within the therapeutic features of psychedelics (CarhartHarris and Nutt [2017]). This is done both at cognitive, neurological and psychological levels. For example, it has been hypothesised that the positive effects of psychedelics on mental disorders like depression is due to the promotion of neural plasticity in the key circuits relevant to treating neuropsychiatric disorders (Ly et al. [2018]), that psychedelics relax the precision weighting of the predictive models encoded in the brain (Cahart-Harris and Friston [2019]) and, on a psychological level, that psychedelics play a crucial role in limiting an agent's belief and revising cognitive biases implicated in mental disorders like depression. This latter claim is often expressed in terms of the idea that psychedelics, in contrast to typical treatment responses, directly address the psychosocial causes of depression, and thus involve a "reset" or "opening up" of a therapeutic window which facilitates insight and emotional release and a revision of a subject's general outlook and life style ([Nutt et al. 2020] p. 34; Cahart-Harris and Nutt [2017]).

\footnotetext{
40 Which is offered will typically will depend on how severe the depressive episode is, with the majority of depressive episodes treated with a combination of medication and psychotherapy (Cleare et al. [2015]; NICE [2016]; Otte et al. [2016].

41 Recent studies suggest there is no meaningful difference between the effects of psychotherapy and pharmacology, although combining these is significantly more effective than one alone (Cuijpers et al. [2009]).

42 These are also being explored as potential treatment options for other mental disorders such as anxiety, addiction and OCD. See (Carhart-Harris and Goodwin [2017]) for a recent review.

43 Similar results were reported in studied with a different psychedelic (ayahuasca) in patients with 'recurrent depression' (Osorio et al. [2015]; Sanches et al. [2016]).
} 
However, if the hypothesis in this paper is true, then there may be further explanatory rationale for this research in the case of depression which is grounded more firmly in the nature of depression itself. That is, if depression does involve a change to one's global state of consciousness, then one can explain the efficacy above via the thought that the ingestion of psychedelics - which presumably, given their primary role in changing one's global state of consciousness - target the very same mechanisms which underly and maintain a central aspect of depression. That is, the the fact that depression involves an altered state of consciousness may play a significant role in explaining why psychedelic treatment is particularly effective in the case of treatment resistant and other forms of depression. Namely, in virtue of the fact that psychedelics act on the same mechanisms implicated in the maintenance of depression ${ }^{44}$. Their ingestion thus may prompt a repair of the general neural circuitry which modulates changes to an individual's global state of consciousness such that an ordinary state of wakeful consciousness is, after a psychedelic 'trip', reinstated or more reliably maintained ${ }^{45}$. The 'reset' often discussed in the context of psychedelic therapy here would thus be grounded in something shared by depression and the psychedelic state which is used to treat it namely, a resetting of one's global state of consciousness.

\section{Conclusion}

Existing attempts to utilise phenomenological work on depression in a psychiatric context are challenged by the fact that the experiential 'shift' characteristic of depression appears to resist analysis in naturalistic terms. The primary aim of this paper has been to establish that there is a viable empirical analysis of the phenomenological shift implicated in depression - on which depression involves a change to one's global state of consciousness - which is explicable in scientific terms. In addition to its ability to explain and account for the distinctive phenomenology of depression, I have suggested that the view on which depression is a disorder of consciousness also has rich explanatory potential in empirical and psychiatric contexts, opening up new conceptual and empirical connections between recent research on global states and the neurobiology of depression and psychedelic psychiatry. The upshot is, in my view, an exciting and conceptually well-motivated hypothesis about the nature of depression which is apt for empirical development.

\footnotetext{
${ }^{44}$ In this sense, the efficacy of psychedelic therapy on depression can be viewed as offering indirect empirical support for my hypothesis.

${ }^{45} \mathrm{Scott}$ and Cahart-Harris ([2019]) have recently suggested that psychedelics ought be developed also as a treatment for post-comatose disorders of consciousness like the PVS. The therapeutic value of this research, given the risks posed by psychedelics for patients, has recently been challenged (Peterson et al. [2019]). The hypothesis here has additional application in this context, suggesting a missing link between psychedelic therapy for depression and the therapeutic potential of psychedelic therapy for treating other disorders of consciousness.
} 


\section{Acknowledgements}

This article has benefited greatly from discussions with numerous people in and outside of philosophy. In particular, I would like to warmly thank Matt Soteriou, Jonathan Birch, Harriet Fagerberg, Matthew Ratcliffe, Andrew Tyler Richmond, Liam Kofi Bright, Geoffrey Whiteley, participants at the London Mind Group and two anonymous referees at BJPS for their helpful, generous and encouraging comments on earlier drafts of this paper. I am also grateful to Eleanor Byrne, Saadhana Manaktala, Anjali Bhat and participants at LSE Philosophy's work-in-progress seminar for several insightful discussions which helped me bring my early idea into paper form. Here I would also like to mention my brother, Matthew Whiteley, whose depression and suicide largely propelled this line of research, and to whom it is dedicated.

Department of Philosophy, Logic and Scientific Method

London School of Economics and Political Science

London, United Kingdom.

c.whiteley@lse.ac.uk 


\section{References}

American Psychiatric Association. Diagnostic and statistical manual of mental disorders (DSM-5®). American Psychiatric Pub, 2013.

Aviram, Liat, and Nirit Soffer-Dudek. "Lucid dreaming: intensity, but not frequency, is inversely related to psychopathology." Frontiers in psychology 9 (2018): 384

Bayne, Tim ; Hohwy, Jakob \& Owen, Adrian M. (2016). Are There Levels of Consciousness? Trends in Cognitive Sciences 20 (6):405-413.

Bayne, T., \& Carter, O. (2018). Dimensions of consciousness and the psychedelic state. Neuroscience of Consciousciousness, 2018(1), niy008. https://doi.org/10.1093/nc/niy008

Beebee, Helen \& Sabbarton-Leary, Nigel (2010). Are psychiatric kinds real? European Journal of Analytic Philosophy 6 (1):11-27.

Bolton, Derek \& Gillett, Grant (2019). The Biopsychosocial Model of Health and Disease: New Philosophical and Scientific Developments. Springer Verlag.

Boly, M., Seth, A. K., Wilke, M., Ingmundson, P., Baars, B., Laureys, S., Edelman, D., \& Tsuchiya, N. (2013). Consciousness in humans and non-human animals: Recent advances and future directions. Frontiers in Psychology, 4, 625. https: //doi.org/10.3389/fpsyg.2013.00625\%20

Boly, M., Massimini, M., Tsuchiya, N., Postle, B. R., Koch, C., \& Tononi, G. (2017). Are the neural correlates of consciousness in the front or in the back of the cerebral cortex? Clinical and neuroimaging evidence. Journal of Neuroscience, 37 (40), 9603-9613. https://doi.org/10.1523/ jneurosci.3218-16.2017

Boyd, Richard. "Homeostasis, species, and higher taxa." Species: New interdisciplinary essays 141 (1999): 185.

Casali, Adenauer G., et al. "A theoretically based index of consciousness independent of sensory processing and behavior." Science translational medicine 5.198 (2013): 198ra105-198ra105.

Carhart-Harris, Robin L., and K. J. Friston. "REBUS and the anarchic brain: toward a unified model of the brain action of psychedelics." Pharmacological reviews 71.3 (2019): 316-344.

Carhart-Harris, Robin L., and Guy M. Goodwin. "The therapeutic potential of psychedelic drugs: past, present, and future." Neuropsychopharmacology 42.11 (2017): 2105-2113.

Carhart-Harris, Robin L., et al. "Psilocybin with psychological support for treatment-resistant depression: six-month follow-up." Psychopharmacology 235.2 (2018): 399-408.

Carhart-Harris, R. L., and D. J. Nutt. "Serotonin and brain function: a tale of two receptors." Journal of Psychopharmacology 31.9 (2017): 1091-1120.

Chalmers, D. J. (2000). What is a neural correlate of consciousness? In T. Metzinger (Ed.), Neural correlates of consciousness (pp. 17-39). MIT Press.

Cleare, A. et al. Evidence-based guidelines for treating depressive disorders with antidepressants: a revision of the 2008 British Association for Psychopharmacology guidelines.

Cooper. R (2020) 'When answers are hard to find, change the question: Asking different causal questions can enable progress, in Davies, Will; Savulescu, Julian \& Roache, Rebecca (eds.) (2020). Psychiatry Reborn: Biopsychosocial Psychiatry in Modern Medicine. Oxford University Press.

Crowther, T. (2018). Experience, dreaming, and the phenomenology of wakeful consciousness. In F. Dorsch, F. MacPherson, \& M. Nida-Rümelin (Eds.), Phenomenal presence (pp. 252-282). Oxford University Press. https://doi.org/10.1093/oso/ 9780199666416.003.0011

Cuijpers, P., van Straten, A., Warmerdam, L.\& Andersson, G. Psychotherapy versus the combination of psychotherapy and pharmacotherapyin the treatment of depression: a meta-analysis.

Depress. Anxiety 26, 279-288 (2009).

Davies, Will \& Roache, Rebecca (2017). Reassessing Biopsychosocial Psychiatry. British Journal of Psychiatry $210(1): 3-5$.

Davies, Will; Savulescu, Julian \& Roache, Rebecca (eds.) (2020). Psychiatry Reborn: Biopsychosocial Psychiatry in Modern Medicine. Oxford University Press.

Fazekas, Peter, and Morten Overgaard. "Multidimensional models of degrees and levels of consciousness." Trends in Cognitive Sciences 20.10 (2016): 715-716. 
Fellowes, Sam. (2021) "The value of categorical Polythetic diagnoses in psychiatry." British Journal for the Philosophy of Science.

Fernandez, Anthony Vincent (2014). Depression as existential feeling or de-situatedness? Distinguishing structure from mode in psychopathology. Phenomenology and the Cognitive Sciences 13 (4):595-612.

Fortier-Davy, M., \& Millière, R. (2020). The multi-dimensional approach to drug-induced states: A commentary on Bayne and Carter's "Dimensions of Consciousness and the Psychedelic State". Neuroscience of Consciousness, 2020(1), niaa004. https://doi.org/10.1093/nc/niaa004\%20

Griffiths RR, Johnson MW, Carducci MA et al (2016) Psilocybin produces substantial and sustained decreases in depression and anxiety in patients with life-threatening cancer: a randomized doubleblind trial. J Psychopharmacol 30:1181-1197

Grob CS, Danforth AL, Chopra GS et al (2011) Pilot study of psilocybin treatment for anxiety in patients with advanced-stage cancer. Arch Gen Psychiatry 68:71-78

Henry, James A., et al. "Underlying mechanisms of tinnitus: review and clinical implications." Journal of the American Academy of Audiology 25.1 (2014): 5-22.

Hoffman, Ginger A., and Peter Zachar. "RDoC's metaphysical assumptions: Problems and promises." Extraordinary science and psychiatry: Responses to the crisis in mental health research (2017): 59-86.

Horwitz, Allan V., and Jerome C. Wakefield. The loss of sadness: How psychiatry transformed normal sorrow into depressive disorder. Oxford University Press, 2007.

Hudetz AG, Liu X, Pillay S, et al. Propofol anesthesia reduces Lempel-Ziv complexity of spontaneous brain activity in rats. Neurosci Lett 2016;628:132-5.

Hyman, Steven E. "The diagnosis of mental disorders: the problem of reification." Annual review of clinical psychology 6 (2010): 155-179.

Insel, Thomas R. "The NIMH research domain criteria (RDoC) project: precision medicine for psychiatry." American Journal of Psychiatry 171.4 (2014): 395-397.

Kendler, K.S \& Gyngell. C (2020). 'Multi-level Interactions and the Dappled Causal World of Psychiatric Disorders. in Davies, Will; Savulescu, Julian \& Roache, Rebecca (eds.) (2020). Psychiatry Reborn: Biopsychosocial Psychiatry in Modern Medicine. Oxford University Press.

Kendler, K. S., Zachar, P., \& Craver, C. (2010). What kinds of things are psychiatric disorders? Psychological Medicine, 41, 1143-1150.

Kendler, Kenneth S. \& Parnas, Josef (eds.) (2012). Philosophical Issues in Psychiatry Ii: Nosology. Oxford University Press

Kennis, Mitzy, et al. "Prospective biomarkers of major depressive disorder: a systematic review and meta-analysis." Molecular psychiatry 25.2 (2020): 321-338.

Kincaid, Harold \& Sullivan, Jacqueline Anne (2014). Classifying Psychopathology: Mental Kinds and Natural Kinds. In Harold Kincaid \& Jacqueline Anne Sullivan (eds.), Classifying Psychopathology: Mental Kinds and Natural Kinds. MIT Press. pp. 1-10.

Kingma, Elselijn (2013). Naturalist accounts of mental disorder. In K. . W. . M. Fulford (ed.), The Oxford Handbook of Philosophy and Psychiatry. Oxford University Press. pp. 363.

Klein, C. (2017). Consciousness, intention, and command-following in the vegetative state. British Journal for the Philosophy of Science, 68(1), 27-54. https://doi.org/10.1093/bjps/axv012\%20

Klein, Colin, Jakob Hohwy, and Tim Bayne. "Explanation in the science of consciousness: From the neural correlates of consciousness (NCCs) to the difference makers of consciousness (DMCs)." Philosophy and the Mind Sciences 1.II (2020).

Koch, Christof, et al. "Posterior and anterior cortex-where is the difference that makes the difference?." Nature Reviews Neuroscience 17.10 (2016): 666-666.

Laureys, Steven (2005). The neural correlate of (un)awareness: Lessons from the vegetative state. Trends in Cognitive Sciences 9 (12):556-559.

Li, Yingjie, et al. "Abnormal EEG complexity in patients with schizophrenia and depression." Clinical Neurophysiology 119.6 (2008): 1232-1241.

Lilienfeld, Scott O. "The Research Domain Criteria (RDoC): An analysis of methodological and conceptual challenges." Behaviour research and therapy 62 (2014): 129-139.

Ly, Calvin, et al. "Psychedelics promote structural and functional neural plasticity." Cell reports 23.11 (2018): 3170-3182. 
Machery, Edouard. "Kinds or Tails?." Extraordinary Science and Psychiatry (2017): 15-36.

Maj, M. (2011). Differentiating depression from ordinary sadness: Contextual, qualitative and pragmatic approaches. World Psychiatry, 11(1), 43-47

Mckilliam, Andy Kenneth (2020). What is a global state of consciousness? Philosophy and the Mind Sciences 1 (II).

McNamara, Patrick, et al. "Impact of REM sleep on distortions of self-concept, mood and memory in depressed/anxious participants." Journal of affective disorders 122.3 (2010): 198-207.

Morales, Jorge \& Lau, Hakwan (2020). The Neural Correlates of Consciousness. In Uriah Kriegel (ed.), The Oxford Handbook of the Philosophy of Consciousness. Oxford University Press. pp. 233-260.

Murphy, Dominic. "Explanation in psychiatry." Philosophy Compass 5.7 (2010): 602-610.

Murphy, Dominic. (2013) "The medical model and the philosophy of science." n K. W. M. Fulford, M. Davies, R. G. T. Gipps, G. Graham, J. Z. Sadler, G. Stanghellini, \& T. Thornton (Eds.), International perspectives in philosophy and psychiatry. The Oxford handbook of philosophy and psychiatry ( $\mathrm{p}$. 966-986). Oxford University Press.

Murphy, D. (2014). Natural kinds in folk psychology and in psychiatry. In H. Kincaid \& J. A. Sullivan (Eds.), Classifying psychopathology: Mental kinds and natural kinds (pp. 105-122). Cambridge: MIT Press.

National Institute for Health and Care Excellence.Depression in adults: recognition and management. NICE https://www.nice.org.uk/guidance/cg90 (2016).

Nutt, David. "Psychedelic drugs_a new era in psychiatry?." Dialogues in clinical neuroscience 21.2 (2019): 139.

Nutt, David, David Erritzoe, and Robin Carhart-Harris. "Psychedelic psychiatry's brave new world." Cell 181.1 (2020): 24-28.

Osório, Flávia de L., et al. "Antidepressant effects of a single dose of ayahuasca in patients with recurrent depression: a preliminary report." Brazilian Journal of Psychiatry 37.1 (2015): 13-20.

O'shaughnessy, Brian. Consciousness and the World. Oxford University Press, 2002.

Otte, Christian, et al. "Major depressive disorder." Nature reviews Disease primers 2.1 (2016): 1-20.

Overgaard, M., \& Overgaard, R. (2010). Neural correlates of contents and levels of consciousness. Frontiers in Psychology, 1, 164. https://doi.org/10.3389/fpsyg.2010.00164\%20

Owen, Matthew, and Mihretu P. Guta. "Physically sufficient neural mechanisms of consciousness." Frontiers in systems neuroscience 13 (2019): 24.

Papineau, D. (1994). Mental disorder, illness and biological disfunction. Royal Institute of

Philosophy Supplements, 37, 73-8

Peterson A, Tagliazucchi E, Weijer C. The ethics of psychedelic research in disorders of consciousness. Neurosci Conscious. 2019;2019(1):niz013. Published 2019 Oct 9. doi:10.1093/nc/ niz013

Radden, Jennifer (2003). Is This Dame Melancholy?: Equating Today's Depression and Past Melancholia. Philosophy, Psychiatry, and Psychology 10 (1):37-52.

Radden, Jennifer \& Varga, Somogy (2013). The epistemological value of depression memoirsi a metaanalysis. In K. W. M. Fulford (ed.), The Oxford Handbook of Philosophy and Psychiatry. Oxford University Press. pp. 99.

Ratcliffe, Matthew (2013). A Bad Case Of The Flu?: The Comparative Phenomenology of Depression and Somatic Illness. Journal of Consciousness Studies 20 (7-8):198-218.

Ratcliffe, Matthew (2015). Experiences of Depression: A Study in Phenomenology. Oxford University Press.

Revonsuo, Antti. Inner presence: Consciousness as a biological phenomenon. Mit Press, 2006.

Richards, C.S and O'Hara M.W (2014). The Oxford handbook of depression and comorbidity. Oxford University Press.

Ross S, Bossis A, Guss J et al (2016) Rapid and sustained symptom reduction following psilocybin treatment for anxiety and depression in patients with life-threatening cancer: a randomized controlled trial. J Psychopharmacol 30:1165-1180 
Rucker, James JH, Jonathan Iliff, and David J. Nutt. "Psychiatry \& the psychedelic drugs. Past, present \& future." Neuropharmacology 142 (2018): 200-218.

Saarinen, Jussi (2018). A critical examination of existential feeling. Phenomenology and the Cognitive Sciences 17 (2):363-374.

Sanches, Rafael Faria, et al. "Antidepressant effects of a single dose of ayahuasca in patients with recurrent depression: a SPECT study." Journal of clinical psychopharmacology 36.1 (2016): 77-81.

Schartner MM, Carhart-Harris RL, Barrett AB, et al. Increased spontaneous MEG signal diversity for psychoactive doses of ketamine, LSD and psilocybin. Sci Rep 2017;7:46421.

Sitt JD, King JR, El Karoui I, et al. Large scale screening of neural signatures of consciousness in patients in a vegetative or min- imally conscious state. Brain 2014;137:2258-70.

Slater, Matthew H. (2013). Are Species Real? An Essay on the Metaphysics of Species. PalgraveMacmillan.

Scott, Gregory, and Robin L. Carhart-Harris. "Psychedelics as a treatment for disorders of consciousness." Neuroscience of consciousness 2019.1 (2019): niz003.

Soteriou, Matthew (2020). Dreams, agency, and judgement. Synthese 197 (12):5319-5334.

Soteriou, Matthew (2019). I-Waking Up and Being Conscious. Aristotelian Society Supplementary Volume 93 (1):111-136.

Szasz, T. S. (1961). The myth of mental illness: Foundations of a theory of personal conduct.

New York: Dell.

Tabb, Kathryn. "Psychiatric progress and the assumption of diagnostic discrimination." Philosophy of Science 82.5 (2015): 1047-1058.

Tabb, Kathryn (2019). Philosophy of psychiatry after diagnostic kinds. Synthese 196 (6):2177-2195.

Tekin, Ş. (2016). Are Mental Disorders Natural Kinds?: A Plea for a New Approach to Intervention in Psychiatry. Philosophy, Psychiatry, \& Psychology, 23(2), 147-163.

Thase, Michael E. "Depression and sleep: pathophysiology and treatment." Dialogues in clinical neuroscience 8.2 (2006): 217.

Tsou, Jonathan Y. (2013). Depression and Suicide are Natural Kinds: Implications for PhysicianAssisted Suicide. International Journal of Law and Psychiatry 36 (5-6):461-470.

Tsou, Jonathan Y. (2016). Natural Kinds, Psychiatric Classification and the History of the DSM. History of Psychiatry 27 (4):406-424.

Tully, Ian (2019). Demarcating depression. Ratio 32 (2):114-121.

Vallat, Raphael, and Perrine Marie Ruby. "Is it a good idea to cultivate lucid dreaming?." Frontiers in psychology 10 (2019): 2585.

Ventham, Elizabeth (2019). Reflective blindness, depression and unpleasant experiences. Analysis 79 (4):684-693.

Voss, Ursula, et al. "Lucid dreaming: a state of consciousness with features of both waking and nonlucid dreaming." Sleep 32.9 (2009): 1191-1200.

Voss, Ursula, and Allan Hobson. What is the state-of-the-art on lucid dreaming?-Recent advances and questions for future research. Open MIND. Frankfurt am Main: MIND Group, 2014.

Windt, Jennifer M. Dreaming: A conceptual framework for philosophy of mind and empirical research. MIT press, 2015.

Wong, Muk (2016). The mood-emotion loop. Philosophical Studies 173 (11):3061-3080.

World Health Organization. International Statistical Classification of Diseases and Related Health Problems (11th Revision). 2020. https://icd.who.int/en. Accessed 2021.

Zeman, Adam, et al. "Phantasia-the psychological significance of lifelong visual imagery vividness extremes." Cortex 130 (2020): 426-440. 